Supporting Information for:

\title{
$\left[\left({ }^{\mathrm{t}} \mathrm{Bu}_{2} \mathrm{PCH}_{2} \mathrm{SiMe}_{2}\right)_{2} \mathrm{~N}\right] \mathrm{RuMe} \mathrm{C}_{2}$ : Synthesis and Reactivity of an Unsaturated Ruthenium Dialkyl Radical Species
}

Michael J. Ingleson, Maren Pink, John C. Huffman, Hongjun Fan and Kenneth G. Caulton* Department of Chemistry \& Molecular Structure Center, Indiana University, Bloomington, Indiana (caulton@indiana.edu).

\section{Contents:}

EPR Spectrum for $\mathrm{PNPRuI}_{2}, 2$

${ }^{1} \mathrm{H}$ NMR Spectra for PNPRuMe 2 , PNPRuI, and PNPRuI ${ }_{2}$

Ball and Stick and ORTEP diagrams of PNPRu(COMe)(CO), 6

Table of crystallographic data for $\mathbf{6}$

Computational Details for the optimized geometry of $\mathbf{1}$ and the SOMO spin density calculation.

S1

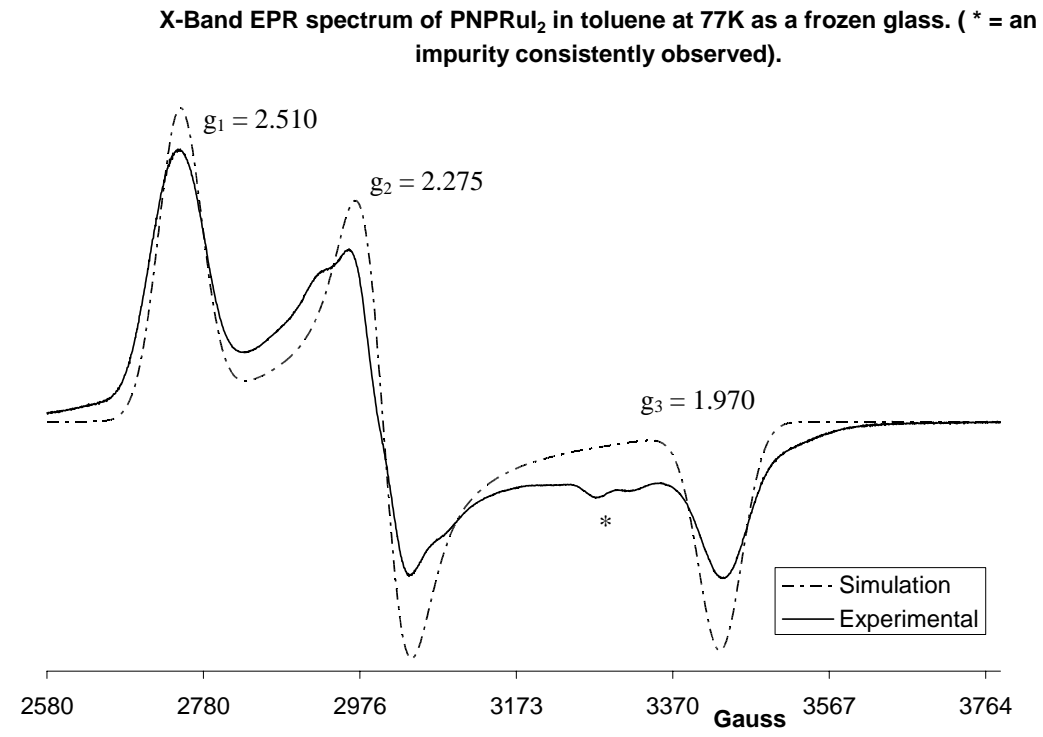


S2: ${ }^{1} \mathrm{H}$ NMR $\left(\mathrm{C}_{6} \mathrm{D}_{6}, 298 \mathrm{~K}\right)$ of PNPRuMe 2

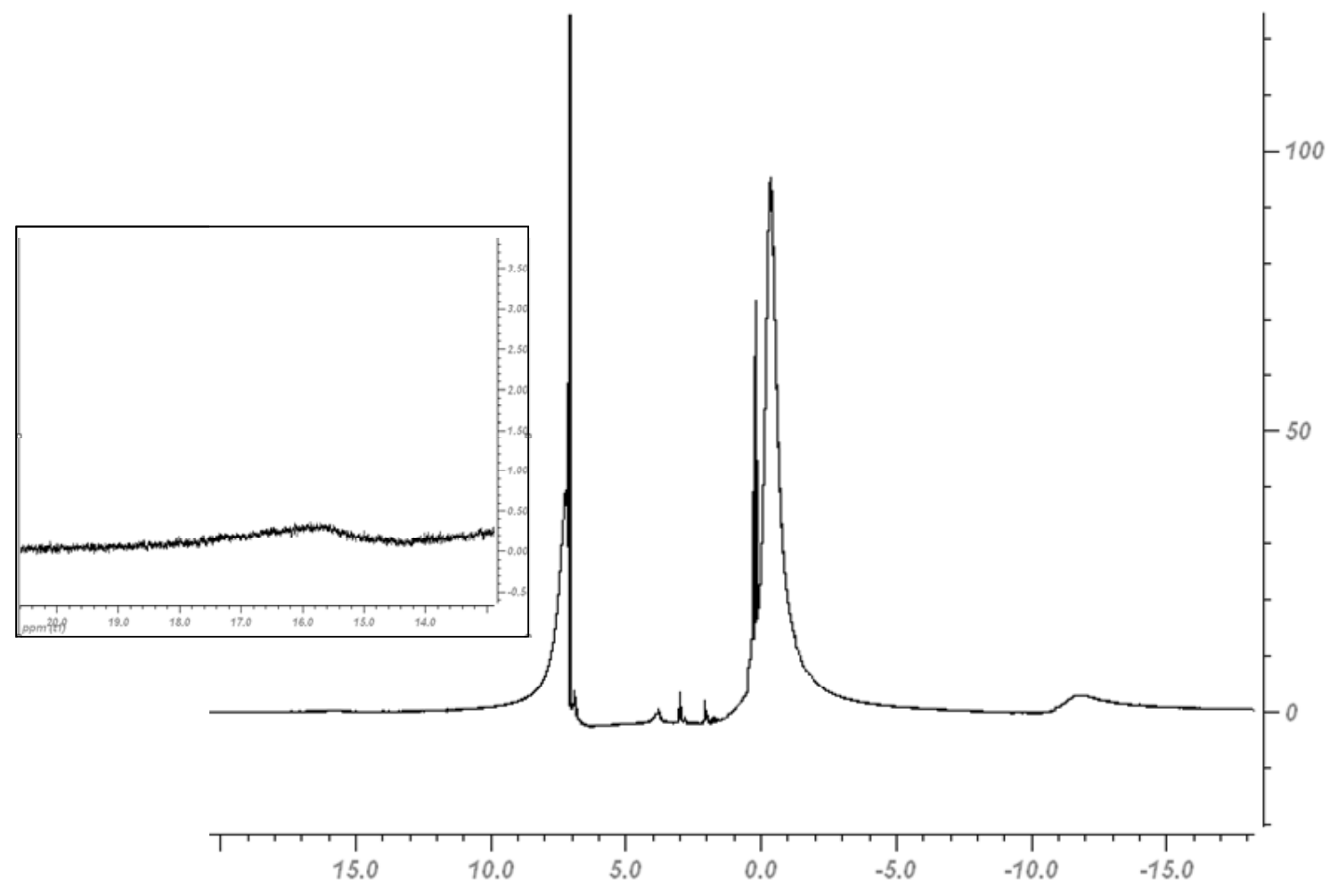


S3: ${ }^{1} \mathrm{H}$ NMR $\left(\mathrm{C}_{6} \mathrm{D}_{6}, 298 \mathrm{~K}\right)$ of PNPRuI
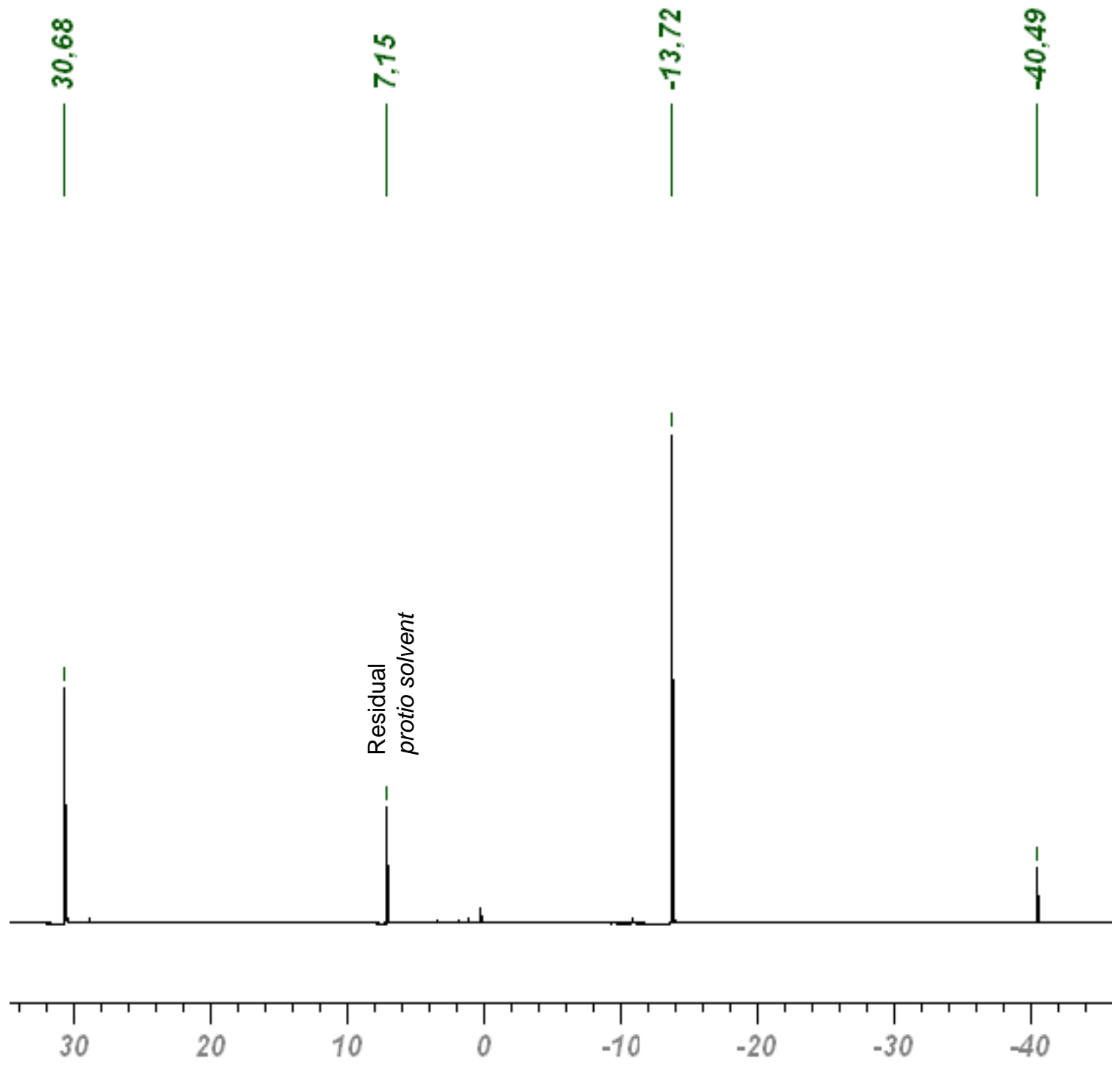
S4: ${ }^{1} \mathrm{H}$ NMR $\left(\mathrm{C}_{6} \mathrm{D}_{6}, 298 \mathrm{~K}\right)$ of PNPRuI 2
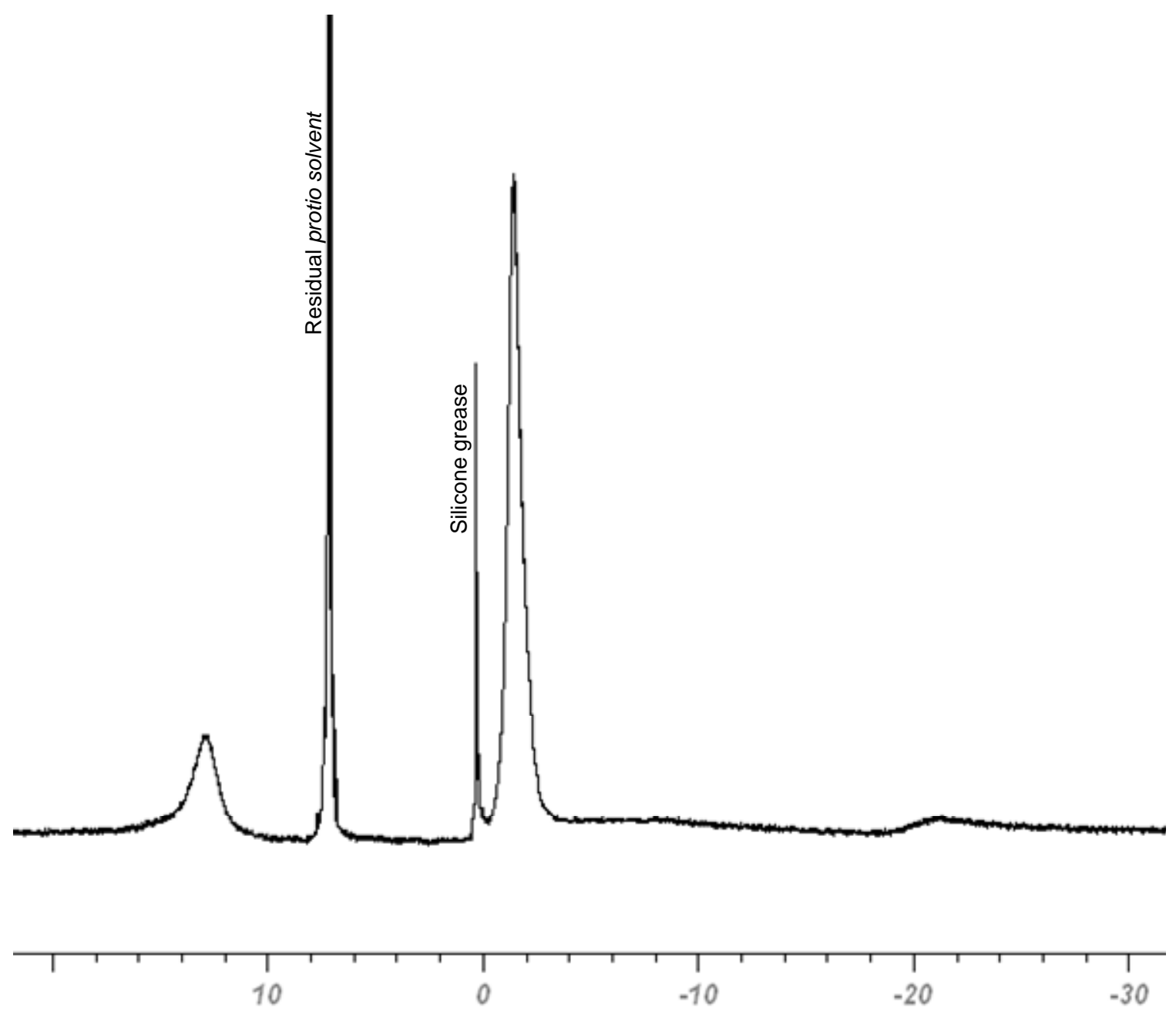


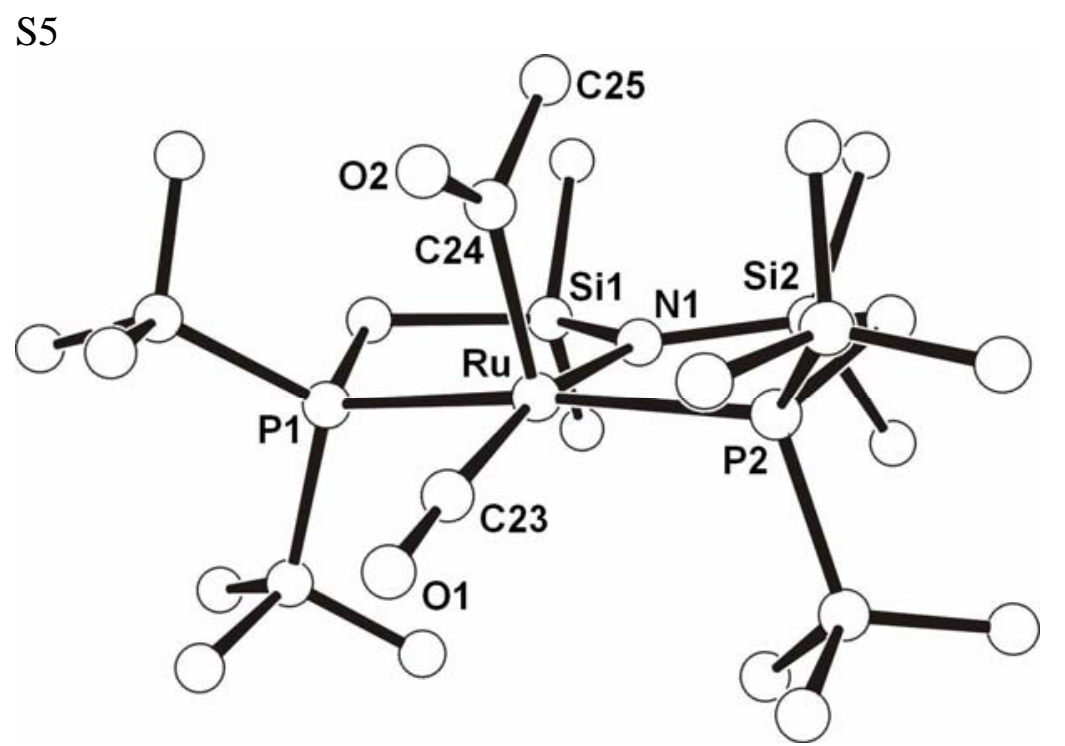

Ball and Stick Representation of PNPRu(COMe)CO, displaying the CO approximately trans to amide and (COMe) trans to the axial site.

The data set collection for PNPRu(COMe)CO, 6 was imperfect with the diffraction pattern showing what appeared to be satellites close to strong spots. With the experimental setup used, they could not be resolved from the main spots. The Fo/Fc lists show unusual discrepancies. The overall displacement of the structure is large. The structure was checked for non-merohedral and pseudo-merohedral twinning; none was found.

The residual electron density is rather large with the two highest peaks of 2.15 (Q1) and 2.06 (Q2) electrons close to Si1 (1.014 Angst.) and C25 (1.30 Angst.), respectively. A disorder model for the PNP ligand including one of the peaks did not improve the R-values and the overall structure. The second highest peak is within bonding distance of C25 and at an angle C24, C25, Q2 of 121.7 degree. Modeling the peak as oxygen atom results in a site occupancy of about 0.33 . Spectroscopic evidence suggests that the structure of the crystal is the one presented in the CIF, excluding any modeling of Q1 and Q2. The current model might be a subcell of a larger modulated structure and supercell. 


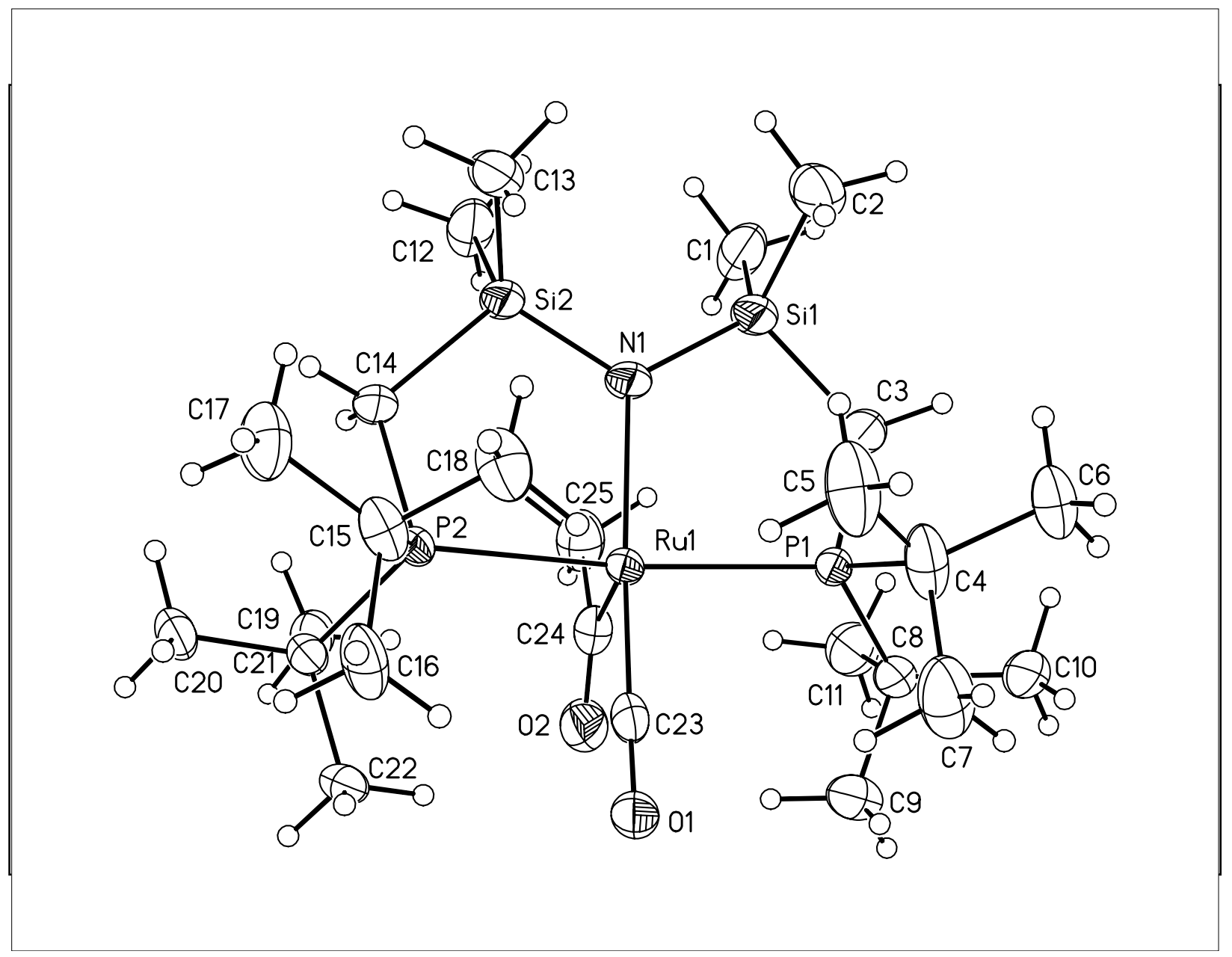

ORTEP view of PNPRu(COMe)Me (50\% probability ellipsoids). 


\section{S7. Crystal data and structure refinement for PNPRu(COMe)CO, 6.}

Empirical formula

Formula weight

Crystal color, shape, size

Temperature

Wavelength

Crystal system, space group

Unit cell dimensions

Volume

$\mathrm{Z}$

Density (calculated)

Absorption coefficient

$\mathrm{F}(000)$

\section{Data collection}

Diffractometer

Theta range for data collection

Index ranges

Reflections collected

Independent reflections

Observed Reflections

Completeness to theta $=26.55^{\circ}$

\section{Solution and Refinement}

Absorption correction

Max. and min. transmission

Solution

Refinement method

Weighting scheme

Data / restraints / parameters

Goodness-of-fit on $\mathrm{F}^{2}$

Final R indices [I $>2$ sigma(I)]

$\mathrm{R}$ indices (all data)

Largest diff. peak and hole
C25 H55 N O2 P2 Ru Si2

620.89

orange block, $0.30 \times 0.20 \times 0.18 \mathrm{~mm}^{3}$

130(2) K

$0.71073 \AA$

Monoclinic, $\mathrm{P} 2{ }_{1} / \mathrm{n}$

$\mathrm{a}=10.1346(11) \AA$

$\alpha=90^{\circ}$.

$\mathrm{b}=29.621(3) \AA$

$\beta=109.978(3)^{\circ}$.

c $=11.2384(12) \AA$

$\gamma=90^{\circ}$.

3170.7(6) $\AA^{3}$

4

$1.301 \mathrm{Mg} / \mathrm{m}^{3}$

$0.692 \mathrm{~mm}^{-1}$

1320

SMART6000 Platform CCD, Bruker

2.25 to $26.55^{\circ}$.

$-12<=\mathrm{h}<=12,-37<=\mathrm{k}<=37,-14<=\mathrm{l}<=13$

25974

$6521[\mathrm{R}(\mathrm{int})=0.0396]$

5729

$98.7 \%$

Semi-empirical from equivalents

0.8855 and 0.8193

Direct methods

Full-matrix least-squares on $\mathrm{F}^{2}$

$\mathrm{w}=\left[\sigma^{2} \mathrm{Fo}^{2}+\mathrm{AP}^{2}+\mathrm{BP}\right]^{-1}$, with

$\mathrm{P}=\left(\mathrm{Fo}^{2}+2 \mathrm{Fc}^{2}\right) / 3, \mathrm{~A}=0.0140, \mathrm{~B}=18.2160$

$6521 / 0 / 315$

1.186

$\mathrm{R} 1=0.0574, \mathrm{wR} 2=0.1303$

$\mathrm{R} 1=0.0654, \mathrm{wR} 2=0.1341$

2.154 and -0.655 e. $\AA^{-3}$

Goodness-of-fit $\left.=\left[\Sigma\left[w\left(\mathrm{~F}_{\mathrm{o}}{ }^{2}-\mathrm{F}_{\mathrm{c}}{ }^{2}\right)^{2}\right] / \mathrm{N}_{\text {observns }}-\mathrm{N}_{\text {params }}\right)\right]^{1 / 2}$, all data.

$\mathrm{R} 1=\Sigma\left(\left|\mathrm{F}_{\mathrm{o}}\right|-\left|\mathrm{F}_{\mathrm{c}}\right|\right) / \Sigma\left|\mathrm{F}_{\mathrm{o}}\right|$.

$w \mathrm{R} 2=\left[\Sigma\left[w\left(\mathrm{~F}_{\mathrm{o}}{ }^{2}-\mathrm{F}_{\mathrm{c}}{ }^{2}\right)^{2}\right] / \Sigma\left[w\left(\mathrm{~F}_{\mathrm{o}}^{2}\right)^{2}\right]\right]^{1 / 2}$. 


\section{S8 Computational Details}

All calculations were carried out using Density Functional Theory as implemented in the Jaguar 5.5 suite $^{1}$ of ab initio quantum chemistry programs. Geometry optimization was performed with the unrestricted B3LYP ${ }^{2-5}$ functional and the 6-31G** basis set. The transition metals were represented using the Los Alamos LACVP** basis $^{6-8}$ that includes relativistic effective core potentials. The spin density and SOMO orbital were calculated by restricted B3LYP on the optimized geometry of unrestricted B3LYP.

\section{References}

(1) Jaguar. 5.5 ed, Schrödinger, L.L.C, Portland, OR, 1991-2003.

(2) Becke, A. D. Phys. Rev. A 1988, 38, 3098.

(3) Becke, A. D. J. Chem. Phys. 1993, 98, 5648.

(4) Lee, C. T.; Yang, W. T.; Parr, R. G. 1988 Phys. Rev. B, 37, 785.

(5) Vosko, S. H.; Wilk, L.; Nusair, M. Can. J. Phys. 1980, 58, 1200.

(6) Hay, P. J.; Wadt, W. R. J. Chem. Phys. 1985, 82, 270.

(7) Hay, P. J.; Wadt, W. R. J. Chem. Phys. 1985, 82, 299.

(8) Wadt, W. R.; Hay, P. J. J. Chem. Phys. 1985, 82, 284. 
Unrestricted B3LYP optimized Structure and energy of $(\mathrm{PNP}) \mathrm{Ru}\left(\mathrm{CH}_{3}\right)_{2}$.

Doublet ground state, $\mathrm{E}(\mathrm{SCF})=-64218.721 \mathrm{eV}$.

$<\mathrm{S} * 2>=0.756$.

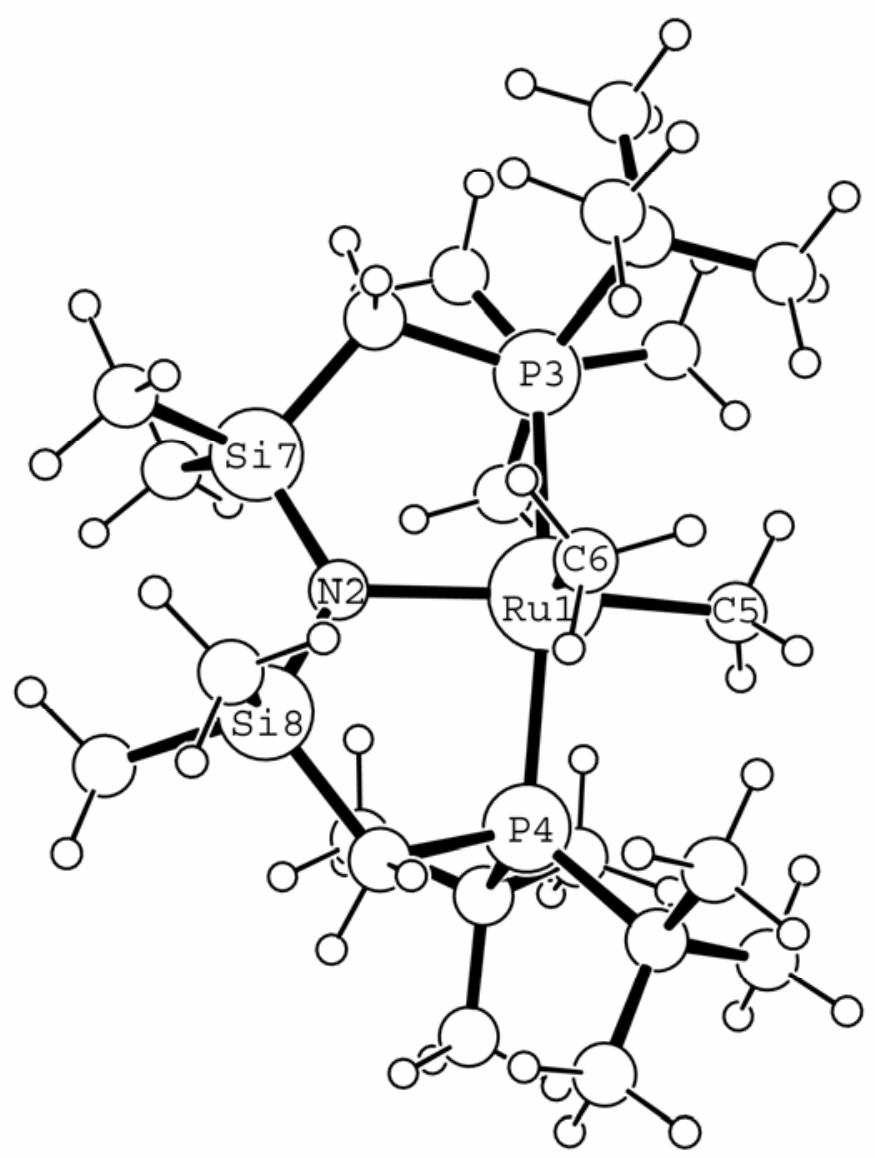

Select bond length (in $\AA$ ) and bond angle (in degree):

$\begin{array}{llll}\text { Ru1-N2: } & 2.191 & \text { C6-Ru1-C5: } & 97.9 \\ \text { Ru1-P3: } & 2.445 & \text { C6-Ru1-N2: } & 93.5 \\ \text { Ru1-P4: } & 2.481 & \text { C6-Ru1-P3: } & 95.0 \\ \text { Ru1-C5: } & 2.160 & \text { C6-Ru1-P4: } & 95.3 \\ \text { Ru1-C6: } & 2.082 & \text { C5-Ru1-N2: } & 168.4 \\ \text { N2-Si7: } & 1.733 & \text { P3-Ru1-P4: } & 168.6 \\ \text { N2-Si8 } & 1.737 & \text { Si7-N2-Ru1: } & 119.7 \\ & & \text { Si8-N2-Ru1: } & 117.8 \\ & & \text { Si7-N2-Si8: } & 121.1\end{array}$


S9: Coordinates:

$\begin{array}{cccc}\mathrm{Ru} & 3.878211377 & 0.419355793 & 2.016464236 \\ \mathrm{P} & 6.024099269 & -0.698545285 & 2.370210236 \\ \mathrm{P} & 1.576005728 & 1.341854057 & 2.083122020 \\ \mathrm{Si} & 3.859053708 & -2.969790827 & 2.291835295 \\ \mathrm{Si} & 1.347096984 & -1.664194123 & 1.234235818 \\ \mathrm{~N} & 2.921479967 & -1.550471007 & 1.958992920 \\ \mathrm{C} & 3.532776200 & -3.749666087 & 3.999831643 \\ \mathrm{H} & 3.586629326 & -3.044636301 & 4.833800382 \\ \mathrm{H} & 4.239750585 & -4.563362193 & 4.205240296 \\ \mathrm{H} & 2.526953395 & -4.185805187 & 4.013991254 \\ \mathrm{C} & 3.621235143 & -4.419085980 & 1.073191889 \\ \mathrm{H} & 2.606477525 & -4.831623958 & 1.093175329 \\ \mathrm{H} & 4.305333113 & -5.237490098 & 1.331657032 \\ \mathrm{H} & 3.837943232 & -4.122388463 & 0.041030220 \\ \mathrm{C} & 5.700551436 & -2.497424409 & 2.076043381 \\ \mathrm{H} & 6.397654396 & -3.162397597 & 2.594944035 \\ \mathrm{H} & 5.864291478 & -2.652259255 & 1.004234380 \\ \mathrm{C} & 7.642878303 & -0.369210589 & 1.351811765 \\ \mathrm{C} & 7.966740156 & 1.138747957 & 1.315819850 \\ \mathrm{H} & 8.839087734 & 1.303263216 & 0.669744144 \\ \mathrm{H} & 8.207643576 & 1.550147631 & 2.297067220 \\ \mathrm{H} & 7.132747547 & 1.713158230 & 0.903892202 \\ \mathrm{C} & 7.402314456 & -0.807997408 & -0.111747087 \\ \mathrm{H} & 8.284849434 & -0.537188873 & -0.704643945 \\ \mathrm{H} & 6.540359980 & -0.310878406 & -0.558970275 \\ \mathrm{H} & 7.272044547 & -1.888658205 & -0.213488436 \\ \mathrm{C} & 8.855469535 & -1.165509846 & 1.874132099 \\ \mathrm{H} & 9.704105961 & -1.018495373 & 1.192915408 \\ \mathrm{H} & 8.652019030 & -2.240831612 & 1.908888996 \\ \mathrm{H} & 9.181076638 & -0.847055699 & 2.866622226 \\ \mathrm{C} & 6.446527658 & -0.597383345 & 4.263992318 \\ \mathrm{C} & 7.365298087 & 0.592182918 & 4.608722511 \\ \mathrm{H} & 7.442723455 & 0.670288398 & 5.701435101 \\ \mathrm{H} & 6.964032938 & 1.540175075 & 4.242094359 \\ \mathrm{H} & 8.380626223 & 0.471523122 & 4.224582595 \\ \mathrm{C} & 7.071559712 & -1.892648083 & 4.818750636 \\ \mathrm{H} & 7.305964396 & -1.750884519 & 5.881953898 \\ \mathrm{H} & 8.001290869 & -2.163438536 & 4.312646003 \\ \mathrm{C} & 5.385982888 & -2.740830243 & 4.750006518 \\ \mathrm{H} & 5.265147386 & -0.344931371 & 6.073958809 \\ \mathrm{H} & 4.362248433 & -1.119872943 & 4.768210303 \\ \mathrm{H} & 1.379403370 & -2.167702209 & -0.602765654\end{array}$

H $\quad 1.737544256 \quad-3.192242448-0.744382454$

H $2.030803445-1.506436376-1.184846774$

H $\quad 0.372326221 \quad-2.110459611 \quad-1.036258701$

C $\quad 0.166475724 \quad-2.905657161 \quad 2.072796524$

H $\quad 0.507679705 \quad-3.936306720 \quad 1.920385200$

H $-0.839981926-2.836518151 \quad 1.640500878$

H $\quad 0.077440605-2.750332704 \quad 3.152665101$

C $\quad 0.522076171 \quad 0.070501896 \quad 1.246036568$

$\mathrm{H} \quad 0.440423587 \quad 0.374946986 \quad 0.195967701$

H $\quad-0.500726010 \quad 0.050647855 \quad 1.639641442$

C $\quad 0.884305681 \quad 1.372518449 \quad 3.893901067$

C $\quad-0.551955769 \quad 1.916118596 \quad 4.019660922$

H $-0.906188925 \quad 1.747052448 \quad 5.045128264$

H $\quad-1.250856271 \quad 1.406139563 \quad 3.349865282$

H $\quad-0.615146865 \quad 2.989984107 \quad 3.831682129$

C $\quad \begin{array}{llll}1.811414557 & 2.193015773 & 4.813633479\end{array}$

H $1.405649564 \quad 2.180195455 \quad 5.833702190$

H $1.903644923 \quad 3.2371541824 .510243452$

H $\quad 2.815515005 \quad 1.767033300 \quad 4.855476093$

C $\quad 0.874375159-0.081581112 \quad 4.413855716$

H $0.660384083 \quad-0.073271153 \quad 5.490386456$

H $\quad \begin{array}{llll}1.826925388 & -0.591945165 & 4.255971567\end{array}$

H $\quad 0.095046284 \quad-0.676559872 \quad 3.932727633$

C $\quad 1.061254462 \quad 2.999446553 \quad 1.209296785$

C $\quad 1.828274997 \quad 3.066911624-0.127642074$

H $\quad 1.562867221 \quad 3.998571857 \quad-0.643940784$

H $\quad 1.564993952 \quad 2.241888882-0.797206167$

H $2.909243854 \quad 3.055834925 \quad 0.019124978$

C $\quad-0.443261351 \quad 3.085535245 \quad 0.878066416$

H $-0.627375566 \quad 4.009233074 \quad 0.313873535$

H -1.0778176463 .1144149351 .763767706$

H $\quad-0.775571647 \quad 2.253780762 \quad 0.250940121$

C $\quad 1.469163573 \quad 4.228058513 \quad 2.046168214$

H $\quad 1.303959308 \quad 5.136734099 \quad 1.452400081$

H $2.525870041 \quad 4.202573531 \quad 2.323198922$

H $\quad 0.873156731 \quad 4.329488801 \quad 2.956523268$

C $\quad 4.699322048 \quad 2.359676757 \quad 2.490942923$

H $5.779361242 \quad 2.381360628 \quad 2.664112274$

H $4.222148032 \quad 2.799934125 \quad 3.376556330$

H $4.495529334 \quad 3.042196265 \quad 1.654829039$

C $4.0690731280 .527938060-0.054352510$

H $4.450320861 \quad-0.427537321 \quad-0.420253877$

H $4.786127055 \quad 1.324511223 \quad-0.281645559$

H $3.121752105 \quad 0.739466889-0.550851875$ 
S10. Mulliken spin density from restricted B3LYP calculation (the order of the atoms is referred to that in the coordinates of S6).

\begin{tabular}{|c|c|c|c|c|c|}
\hline Atom & $\mathrm{Ru}$ & $P$ & $P$ & $\mathrm{Si}$ & $\mathrm{Si}$ \\
\hline Spin & $\odot .84434$ & $\odot .0 \odot \odot 51$ & $\odot . \odot \odot \odot 48$ & $\odot .00777$ & ๑. 00861 \\
\hline Atom & $\mathrm{N}$ & C & $\mathrm{H}$ & H & $\mathrm{H}$ \\
\hline Spin & $\odot .07597$ & ๑. . 00172 & ๑. ๑๑९७5 & $\odot .00031$ & $-\odot .00001$ \\
\hline Atom & C & $\mathrm{H}$ & $\mathrm{H}$ & $\mathrm{H}$ & C \\
\hline Spin & 0.00311 & $-\odot .0 \odot \odot ० 7$ & ๑.๑००65 & $-\odot .00005$ & ๑. 00016 \\
\hline Atom & $\mathrm{H}$ & $\mathrm{H}$ & C & C & $\mathrm{H}$ \\
\hline Spin & $\odot . \odot \odot \odot \odot \odot$ & $-\odot .0 \odot \odot \odot 2$ & ๑. $\odot \odot \odot 17$ & ๑. ๑๑९९૩ & $\odot . \odot \odot \odot \odot \odot$ \\
\hline Atom & $\mathrm{H}$ & $\mathrm{H}$ & C & $\mathrm{H}$ & $\mathrm{H}$ \\
\hline Spin & $\odot . ๑ \odot \odot \odot \odot ~$ & $\odot .0 \odot \odot \odot \odot$ & ๑. ๑๑९७2 & ๑. ๑๑९७९ & $\odot .0 \odot \odot \odot \odot$ \\
\hline Atom & $\mathrm{H}$ & C & $\mathrm{H}$ & H & H \\
\hline Spin & $\odot . \odot \odot \odot \odot \odot$ & $\odot . \odot \odot \odot \odot 4$ & 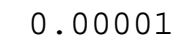 & ๑. ๑๑९७९ & $\odot . \odot \odot \odot \odot \odot$ \\
\hline Atom & C & C & $\mathrm{H}$ & $\mathrm{H}$ & $\mathrm{H}$ \\
\hline Spin & ๑.๑०७०1 & $\odot . ๑ \odot \odot \odot \odot ~$ & ๑ . ๑๑९७९ & $-\odot .0 \odot \odot \odot 1$ & $\odot . \odot \odot \odot \odot \odot$ \\
\hline Atom & C & $\mathrm{H}$ & $\mathrm{H}$ & $\mathrm{H}$ & C \\
\hline Spin & $\odot . \odot \odot \odot \odot \odot$ & 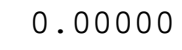 & ๑.๑๑७७९ & $\odot . \odot \odot \odot \odot \odot ~$ & $\odot . \odot \odot \odot 2 \odot$ \\
\hline Atom & $\mathrm{H}$ & $\mathrm{H}$ & $\mathrm{H}$ & C & $\mathrm{H}$ \\
\hline Spin & 0.00004 & ๑.00002 & -0.00007 & ๑. 00399 & -0.00009 \\
\hline Atom & $\mathrm{H}$ & $\mathrm{H}$ & C & $\mathrm{H}$ & $\mathrm{H}$ \\
\hline Spin & $\odot . \odot \odot \odot \odot 2$ & $\odot . \odot \odot \odot 97$ & $\odot . \odot \odot \odot \odot 1$ & $\odot . \odot \odot \odot \odot 1$ & $\odot . \odot \odot \odot \odot \odot$ \\
\hline Atom & $\mathrm{H}$ & C & $\mathrm{H}$ & $\mathrm{H}$ & C \\
\hline Spin & 0.00004 & ๑.00066 & ๑.00002 & ๑. .0000० & 0.00004 \\
\hline Atom & C & $\mathrm{H}$ & $\mathrm{H}$ & $\mathrm{H}$ & C \\
\hline Spin & $\odot . \odot \odot \odot \odot \odot$ & 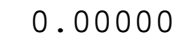 & $\odot . \odot \odot \odot \odot \odot$ & $\odot . \odot \odot \odot \odot 1$ & $\odot . \odot \odot \odot \odot 2$ \\
\hline Atom & $\mathrm{H}$ & $\mathrm{H}$ & H & C & $\mathrm{H}$ \\
\hline Spin & ๑.०००००० & 0.00001 & $\odot . \odot \odot \odot \odot \odot ~$ & $\odot .0 \odot \odot 1 \odot$ & 0.00002 \\
\hline Atom & $\mathrm{H}$ & $\mathrm{H}$ & C & C & $\mathrm{H}$ \\
\hline Spin & $\odot .0 \odot \odot 33$ & $-\odot . \odot \odot \odot \odot 2$ & $\odot .00 \odot \odot 4$ & $\odot .00005$ & ๑. 00001 \\
\hline Atom & $\mathrm{H}$ & $\mathrm{H}$ & C & H & $\mathrm{H}$ \\
\hline Spin & $\odot .00001$ & $\odot .000 \odot 2$ & $\odot . \odot \odot \odot \odot 3$ & $\odot .0 \odot \odot \odot 1$ & $\odot . \odot \odot \odot \odot \odot$ \\
\hline Atom & $\mathrm{H}$ & C & $\mathrm{H}$ & $\mathrm{H}$ & $\mathrm{H}$ \\
\hline Spin & $\odot . \odot \odot \odot \odot \odot$ & $\odot .0 \odot \odot \odot 2$ & $\odot . \odot \odot \odot \odot \odot$ & $-\odot .0 \odot \odot \odot 1$ & $\odot .0 \odot \odot \odot 1$ \\
\hline Atom & C & H & H & $\mathrm{H}$ & C \\
\hline Spin & ๑.01533 & ๑.00052 & ๑. 00555 & $\odot .00829$ & $0.0 \odot 477$ \\
\hline Atom & $\mathrm{H}$ & $\mathrm{H}$ & $\mathrm{H}$ & & \\
\hline Spin & $\odot .00542$ & 0.00925 & 0.00054 & & \\
\hline
\end{tabular}


S11. The picture of SOMO from restricted B3LYP calculation (the contour line is 0.07).

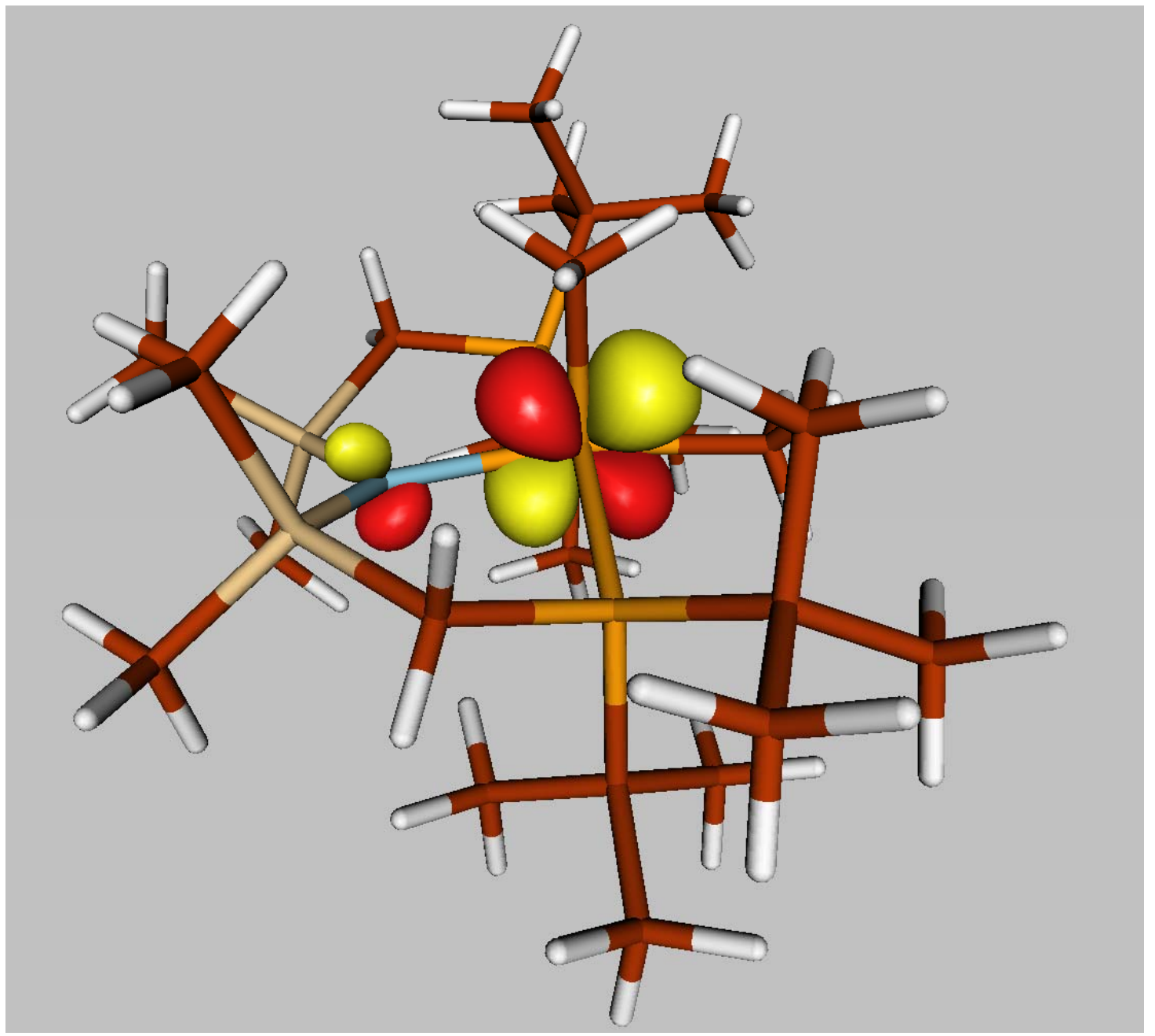

